\title{
A generalization of strongly Gorenstein projective modules
}

\section{Driss Bennis and Najib Mahdou}

\author{
Department of Mathematics, Faculty of Science and Technology of Fez, \\ Box 2202, University S. M. Ben Abdellah Fez, Morocco, \\ driss_bennis@hotmail.com \\ mahdou@hotmail.com
}

\begin{abstract}
This paper generalize the idea of the authors in J. Pure Appl. Algebra 210 (2007) 437-445. Namely, we define and study a particular case of Gorenstein projective modules. We investigate some change of rings results for this new kind of modules. Examples over not necessarily Noetherian rings are given.
\end{abstract}

Keywords: ( $n$-Strongly) Gorenstein projective modules; change of rings results.

2000 Mathematics Subject Classification. 13D05, 13D07, 16E05, 16E10, 16E30

\section{Introduction}

Throughout this paper, $R$ denotes a non-trivial associative ring with identity, and all modules are, if not specified otherwise, left $R$-modules. For an $R$-module $M$, we use $\operatorname{pd}_{R}(M)$ to denote the classical projective dimension of $M$. It is convenient to use "local" to refer to (not necessarily Noetherian) rings with a unique maximal ideal.

We assume that the reader is familiar with the Gorenstein homological dimension theory. Some references are [6, 17, 8, 13. Nevertheless, it is convenient to give a brief history of the Gorenstein dimension theory.

In the sixties, Auslander and Bridger introduced an homological dimension, called G-dimension, for finitely generated modules over Noetherian rings 1, 2. Several decades later, this homological dimension was extended, by Enochs et al. [9, 10, 12, to Gorenstein projective dimension of modules that are not necessarily finitely generated and over not necessarily Noetherian rings. And, dually, they defined the Gorenstein injective dimension. Then, to complete the analogy with the classical homological dimensions, Enochs et al. 11] introduced the Gorenstein flat dimension.

In the last years, the Gorenstein homological dimensions have become a vigorously active area of research (see 6] and 8] for more details). In 2004, Holm 13] generalized several results which are already obtained over Noetherian rings to associative rings. Recently, in [3], the authors introduced a particular case of Gorenstein projective, injective, and flat modules, which are defined, respectively, as follows:

*Journal of Algebra and Its Applications, To appear 
Definition $1.1([3]) \quad$ - An $R$-module $M$ is said to be strongly Gorenstein projective (SGprojective for short), if there exists an exact sequence of projective modules

$$
\mathbf{P}=\cdots \stackrel{f}{\longrightarrow} P \stackrel{f}{\longrightarrow} P \stackrel{f}{\longrightarrow} P \stackrel{f}{\longrightarrow} \cdots
$$

such that $M \cong \operatorname{Im}(f)$ and such that $\operatorname{Hom}_{R}(-, Q)$ leaves the sequence $\mathbf{P}$ exact whenever $Q$ is a projective $R$-module.

- The strongly Gorenstein injective (SG-injective for short) modules are defined dually.

- An $R$-module $M$ is said to be strongly Gorenstein flat (SG-flat for short), if there exists an exact sequence of flat $R$-modules

$$
\mathbf{F}=\cdots \stackrel{f}{\longrightarrow} F \stackrel{f}{\longrightarrow} F \stackrel{f}{\longrightarrow} F \stackrel{f}{\longrightarrow} \cdots
$$

such that $M \cong \operatorname{Im}(f)$ and such that $I \otimes_{R}-$ leaves the sequence $\mathbf{F}$ exact whenever $I$ is an injective right $R$-module.

It is proved that the class of all strongly Gorenstein projective modules is an intermediate class between the ones of projective modules and Gorenstein projective modules [3, Proposition 2.3]; i.e., we have the following inclusions

$$
\begin{aligned}
\text { \{projective modules }\} & \subseteq\{S G \text {-projective modules }\} \\
& \subseteq\{G \text {-projective modules }\}
\end{aligned}
$$

which are strict by [3, Examples 2.5 and 2.13]. The principal role of the strongly Gorenstein projective, injective, and flat modules is to give a simple characterization of Gorenstein projective, injective, and flat modules, respectively (see [3. Theorems 2.7 and 3.5]). For example, we have, in the Gorenstein projective case, that a module is Gorenstein projective if and only if it is a direct summand of a strongly Gorenstein projective module*. The strongly Gorenstein modules confirm that there is an analogy between the notion of "Gorenstein projective, injective, and flat modules" and the notion of the usual "projective, injective, and flat modules". This is, in fact obtained, because the strongly Gorenstein projective, injective, and flat modules have simpler characterizations than their Gorenstein correspondent modules [3, Propositions 2.9 and 3.6]. For instance, a module $M$ is strongly Gorenstein projective if and only if there exists a short exact sequence $0 \rightarrow M \rightarrow P \rightarrow M \rightarrow 0$, where $P$ is a projective module, such that the short sequence $0 \rightarrow \operatorname{Hom}(M, Q) \rightarrow \operatorname{Hom}(P, Q) \rightarrow \operatorname{Hom}(M, Q) \rightarrow 0$ is exact for any projective module $Q$.

The aim of this paper is to generalize the notion of "strongly Gorenstein projective modules" to $n$-strongly Gorenstein projective modules (Definition 2.1). We generalize some results of [3] (see Theorem 2.8 and Proposition 2.2), we establish some change of rings results (Theorem 2.10 and Corollary 2.11), and we give some examples of $n$-strongly Gorenstein projective modules over not necessarily Noetherian rings (see Examples 2.4 and 2.6).

\section{$2 n$-Strongly Gorenstein projective modules}

In this section, we introduce and study $n$-strongly Gorenstein projective modules which are defined as follows:

* In [3] the rings are assumed to be commutative. However, one can show easily that this assumption is not necessary for some results such as the principal one [3. Theorem 2.7] and [3. Proposition 2.9], which we need in this paper. 
Definition 2.1 Let $n$ be a positive integer. An $R$-module $M$ is said to be $n$-strongly Gorenstein projective ( $n$-SG-projective for short), if there exists an exact sequence of $R$-modules

$$
0 \rightarrow M \rightarrow P_{n} \rightarrow \cdots \rightarrow P_{1} \rightarrow M \rightarrow 0
$$

where each $P_{i}$ is projective, such that $\operatorname{Hom}_{R}(-, Q)$ leaves the sequence exact whenever $Q$ is a projective $R$-module.

Consequently, the 1-strongly Gorenstein projective modules are just the strongly Gorenstein projective modules (by [3, Proposition 2.9]).

Recall that a Gorenstein projective module is projective if and only if it has finite projective dimension [13, Proposition 2.27]. For the $n$-strongly Gorenstein projective modules, we have the following result, which is a generalization of [3, Corollary 2.11].

Proposition 2.2 Let $n$ be a positive integer. An n-strongly Gorenstein projective module is projective if and only if it has finite flat dimension.

Proof. The "only if" part is trivial.

We prove the "if" part. Let $M$ be an $n$-strongly Gorenstein projective module with finite flat dimension. Then, there exists an exact sequence of modules

$$
(\alpha) \quad 0 \rightarrow M \rightarrow P_{n} \rightarrow \cdots \rightarrow P_{1} \rightarrow M \rightarrow 0,
$$

where each $P_{i}$ is projective, and, by standard arguments, $M$ is flat. We decompose the above sequence $(\alpha)$ into short exact sequences

$$
\left(\alpha_{i}\right) \quad 0 \rightarrow H_{i} \rightarrow P_{i} \rightarrow H_{i-1} \rightarrow 0
$$

where $H_{n}=M=H_{0}$ and $H_{i}=\operatorname{Ker}\left(P_{i} \rightarrow H_{i-1}\right)$ for $i=n-1, \ldots, 1$. It is evident that each $H_{i}$ is flat, so is the finite direct sum $H=\bigoplus_{1 \leq i \leq n} H_{i}$. Now, by adding the short exact sequences $\left(\alpha_{i}\right)$, we obtain the following short exact sequence:

$$
0 \rightarrow H \rightarrow P \rightarrow H \rightarrow 0
$$

where $P=\bigoplus_{1 \leq i \leq n} P_{i}$. Finally, 4, Theorem 2.5] gives the desired result.

Corollary 2.3 If $R$ has finite weak dimension, then the class of all projective $R$-modules and the class of all Gorenstein projective $R$-modules are the same class.

Proof. Apply Proposition 2.2 and [3, Theorem 2.7].

The following gives an example, over a not necessarily Noetherian ring, of a 1-strongly Gorenstein projective module which is not projective.

Example 2.4 Let $R$ be a local ring and consider the ring $S=R[[X]] /\left(X^{2}\right)$. Denote by $\bar{X}$ the residue class in $S$ of $X$. Then, the ideal $(\bar{X})$ is strongly Gorenstein projective but it is not projective. 
Proof. First, since $R$ is local, $S$ is also local, and so the ideal $(\bar{X})$ is not projective $\left(\right.$ since $\left.(\bar{X})^{2}=0\right)$. Now, we prove that $(\bar{X})$ is strongly Gorenstein projective.

Let $x$ be the homothety given by multiplication by $\bar{X}$. We have the exact sequence:

$$
\cdots \longrightarrow S \stackrel{x}{\longrightarrow} S \stackrel{x}{\longrightarrow} S \longrightarrow \cdots
$$

such that $\operatorname{Ker} x=\operatorname{Im} x=(\bar{X})$.

Since $S$ is local, every projective $S$-module is free. Then, consider a free $S$-module $S^{(I)}$, where $I$ is an index set, and applying the functor $\operatorname{Hom}_{S}\left(-, S^{(I)}\right)$ to the above sequence, we get the following sequence:

$$
\cdots \longrightarrow \operatorname{Hom}\left(S, S^{(I)}\right) \stackrel{\operatorname{Hom}_{S}\left(x, S^{(I)}\right)}{\longrightarrow} \operatorname{Hom}\left(S, S^{(I)}\right) \longrightarrow \cdots
$$

By the natural isomorphism $\operatorname{Hom}\left(S, S^{(I)}\right) \stackrel{\cong}{\longrightarrow} S^{(I)}$, we get the following commutative diagram:

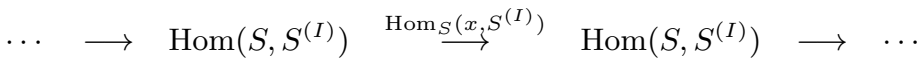

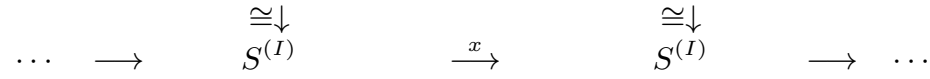

Evidently, the lower sequence in the diagram above is exact, then so is the upper sequence, which means that the ideal $(\bar{X})$ is strongly Gorenstein projective.

Proposition 2.5 Let $n$ be a positive integer.

1. Every strongly Gorenstein projective module is n-strongly Gorenstein projective.

2. Every n-strongly Gorenstein projective module is Gorenstein projective.

Proof. 1. Let $M$ be a strongly Gorenstein projective module. Then, there exists, by [3, Proposition 2.9], a short exact sequence: $0 \longrightarrow M \stackrel{f}{\longrightarrow} P \stackrel{g}{\longrightarrow} M \longrightarrow 0$, where $P$ is a projective module, such that the short sequence $0 \rightarrow \operatorname{Hom}(M, Q) \rightarrow \operatorname{Hom}(P, Q) \rightarrow \operatorname{Hom}(M, Q) \rightarrow 0$ is exact for any projective module $Q$. Thus, we get an exact sequence:

$$
0 \longrightarrow M \stackrel{f}{\longrightarrow} P \stackrel{f \circ g}{\longrightarrow} \ldots \stackrel{f \circ g}{\longrightarrow} P \stackrel{g}{\longrightarrow} M \longrightarrow 0,
$$

such that $\operatorname{Hom}(-, Q)$ leaves the sequence exact whenever $Q$ is a projective module. This, shows that $M$ is $n$-strongly Gorenstein projective for every positive integer $n$.

2. Let $M$ be a $n$-strongly Gorenstein projective module. Then, there exists an exact sequence

$$
0 \longrightarrow M \stackrel{f_{n+1}}{\longrightarrow} P_{n} \stackrel{f_{n}}{\longrightarrow} \cdots \stackrel{f_{2}}{\longrightarrow} P_{1} \stackrel{f_{1}}{\longrightarrow} M \longrightarrow 0,
$$

where each $P_{i}$ is a projective module, such that $\operatorname{Hom}(-, Q)$ leaves the sequence exact whenever $Q$ is a projective module. Thus, we get an exact sequence:

$$
\cdots \stackrel{f_{2}}{\longrightarrow} P_{1} \stackrel{f_{n+1} \circ f_{1}}{\longrightarrow} P_{n} \stackrel{f_{n}}{\longrightarrow} \cdots \stackrel{f_{2}}{\longrightarrow} P_{1} \stackrel{f_{n+1} \circ f_{1}}{\longrightarrow} P_{n} \stackrel{f_{n}}{\longrightarrow} \cdots
$$

such that $\operatorname{Hom}(-, Q)$ leaves the sequence exact whenever $Q$ is a projective module. This shows that $M$ is Gorenstein projective.

The following is an extension of [3. Example 2.13], such that we give an example of 2-strongly Gorenstein projective modules, over a not necessarily Noetherian ring, which are not 1-strongly Gorenstein projective. 
Example 2.6 Let $R$ be a local ring and consider the ring $S=R[[X, Y]] /(X Y)$. Then:

1. The two ideals $(\bar{X})$ and $(\bar{Y})$ are 2-strongly Gorenstein projective, where $\bar{X}$ and $\bar{Y}$ are the residue classes in $S$ of $X$ and $Y$, respectively.

2. $(\bar{X})$ and $(\bar{Y})$ are not strongly Gorenstein projective.

Proof. The proof of the statement (1) is similar to the one of Example 2.4 We only need to note that we have two exact sequences:

$$
\begin{aligned}
& 0 \longrightarrow(\bar{X}) \longrightarrow R \stackrel{y}{\longrightarrow} R \stackrel{x}{\longrightarrow}(\bar{X}) \rightarrow 0 \\
& 0 \longrightarrow(\bar{Y}) \longrightarrow R \stackrel{x}{\longrightarrow} R \stackrel{y}{\longrightarrow}(\bar{Y}) \longrightarrow 0
\end{aligned}
$$

where $x$ and $y$ are the multiplications by $\bar{X}$ and $\bar{Y}$, respectively.

The proof of the statement (2) is the same as the one of [3, Example $2.13(2)$ ].

Obviously, any example of an injective module which is not projective is an example of (strongly) Gorenstein injective module which is not (strongly) Gorenstein projective. From the following, which is a generalization of [14, Exercise 4.2, p. 115], we can construct an example of an ( $n$-strongly) Gorenstein injective module which is neither injective nor ( $n$-strongly) Gorenstein projective.

Proposition 2.7 If $R$ is an integral domain but not a field, an $R$-module that is simultaneously Gorenstein projective and Gorenstein injective must be 0 .

Proof. Assume that an $R$-module $M$ is simultaneously Gorenstein projective and Gorenstein injective. Then, as a Gorenstein projective $R$-module, $M$ embeds in a projective $R$-module, and so it is torsion-free (since $R$ is an integral domain). On the other hand, as a Gorenstein injective $R$-module, $M$ is a quotient of an injective $R$-module, and so it is divisible (by [14 Exercise 3.14, p. 69]). Thus, by [14, Exercise 3.19, p. 70], $M$ is an injective $R$-module, then it is also projective (since it embeds in a projective $R$-module). Finally, by [14, Exercise 4.2, p. 115], $M$ must be 0 .

The next result is a characterization of $n$-strongly Gorenstein projective modules. It is a generalization of [3, Proposition 2.9].

Theorem 2.8 For any module $M$ and any positive integer $n$, the following are equivalent:

1. $M$ is n-strongly Gorenstein projective;

2. There exists an exact sequence $0 \rightarrow M \rightarrow P_{n} \rightarrow \cdots \rightarrow P_{1} \rightarrow M \rightarrow 0$, where each $P_{i}$ is a projective module, such that $\operatorname{Hom}\left(-, Q^{\prime}\right)$ leaves the sequence exact whenever $Q^{\prime}$ is a module with finite projective dimension;

3. There exists an exact sequence $0 \rightarrow M \rightarrow P_{n} \rightarrow \cdots \rightarrow P_{1} \rightarrow M \rightarrow 0$, where each $P_{i}$ is a projective module, and there exists a positive integer $j$ such that $\operatorname{Ext}^{j+1}(M, Q)=\operatorname{Ext}^{j+2}(M, Q)=$ $\cdots=\operatorname{Ext}^{j+n}(M, Q)=0$ for any projective module $Q$;

4. There exists an exact sequence $0 \rightarrow M \rightarrow P_{n} \rightarrow \cdots \rightarrow P_{1} \rightarrow M \rightarrow 0$, where each $P_{i}$ is a projective module, and there exists a positive integer $j$ such that $\operatorname{Ext}^{j+1}\left(M, Q^{\prime}\right)=$ $\operatorname{Ext}^{j+2}\left(M, Q^{\prime}\right)=\cdots=\operatorname{Ext}^{j+n}\left(M, Q^{\prime}\right)=0$ for any module $Q^{\prime}$ with finite projective dimension. 
Proof. $1 \Leftrightarrow 2$. Obviously the second condition is stronger than the first, then only the implication $1 \Rightarrow 2$ merits a proof. So, let $M$ be an $n$-strongly Gorenstein projective module. Then, there exists an exact sequence

$$
\mathbf{P}=0 \rightarrow M \rightarrow P_{n} \rightarrow \cdots \rightarrow P_{1} \rightarrow M \rightarrow 0,
$$

where each $P_{i}$ is a projective module, such that $\operatorname{Hom}(-, Q)$ leaves the sequence exact whenever $Q$ is a projective module. Let $Q^{\prime}$ be a module with $\operatorname{pd}\left(Q^{\prime}\right)=m<\infty$ and consider a short exact sequence $0 \rightarrow K \rightarrow F \rightarrow Q^{\prime} \rightarrow 0$, where $F$ is a free module, then $\operatorname{pd}(K)=m-1$. Thus,

$$
0 \rightarrow \operatorname{Hom}(\mathbf{P}, K) \rightarrow \operatorname{Hom}(\mathbf{P}, F) \rightarrow \operatorname{Hom}\left(\mathbf{P}, Q^{\prime}\right) \rightarrow 0
$$

is an exact sequence of complexes (the exactness of $\operatorname{Hom}(\mathbf{P}, F) \rightarrow \operatorname{Hom}\left(\mathbf{P}, Q^{\prime}\right) \rightarrow 0$ follows by 13 , Proposition 2.3], since $M$ is $n$-strongly Gorenstein projective then Gorenstein projective). Now, by inductive hypothesis, the complexes $\operatorname{Hom}(\mathbf{P}, K)$ and $\operatorname{Hom}(\mathbf{P}, F)$ are exact, then so is $\operatorname{Hom}\left(\mathbf{P}, Q^{\prime}\right)$ (by [14, Theorem 6.3]), as desired.

To prove the equivalences $1 \Leftrightarrow 3$ and $2 \Leftrightarrow 4$, it suffices to apply the following remark. If there exists an exact sequence of the form:

$$
0 \rightarrow M \rightarrow P_{n} \rightarrow \cdots \rightarrow P_{1} \rightarrow M \rightarrow 0
$$

where each $P_{i}$ is a projective module, we get $\operatorname{Ext}^{i}(M, G) \cong \operatorname{Ext}^{n+i}(M, G)$ for all $i \geq 1$ and all modules $G$ (from [14, Theorem 9.4]). This implies that $\operatorname{Ext}^{i}(M, G)=0$ for all $i \geq 1$ whenever $n$ successive terms of $\operatorname{Ext}(M, G)$ vanish.

Recall that a ring $R$ is called Iwanaga-Gorenstein ring (or simply a Gorenstein ring), if $R$ is both left and right Noetherian and if $R$ has finite self-injective dimension on both the left and the right 8. Definition 9.1.1]. Over Gorenstein rings, $n$-strongly Gorenstein projective modules have the following characterization:

Corollary 2.9 If $R$ is a Gorenstein ring, then an $R$-module $M$ is n-strongly Gorenstein projective if and only if there exits an exact sequence of the form $0 \rightarrow M \rightarrow P_{n} \rightarrow \cdots \rightarrow P_{1} \rightarrow M \rightarrow 0$, where each $P_{i}$ is a projective $R$-module.

Proof. Simply apply Theorem 2.8 and the fact that over a Gorenstein ring every projective module has finite injective dimension [8, Proposition 9.1.7].

Now, we give change of rings results.

Theorem 2.10 Let $R \rightarrow S$ be a homomorphism of commutative rings with $\operatorname{pd}_{R}(S)<\infty$. If $M$ is an $n$-strongly Gorenstein projective $R$-module $(n \geq 1)$, then $S \otimes_{R} M$ is an n-strongly Gorenstein projective $S$-module.

Proof. Let $M$ be an $n$-strongly Gorenstein projective $R$-module. Then, there exists, by Theorem 2.8 and its proof, an exact sequence of $R$-modules $0 \rightarrow M \rightarrow P_{n} \rightarrow \cdots \rightarrow P_{1} \rightarrow M \rightarrow 0$, where each $P_{i}$ is projective, and $\operatorname{Ext}_{R}^{i}\left(M, Q^{\prime}\right)=0$ for any $i \geq 1$ and for any $R$-module $Q^{\prime}$ with finite projective dimension.

As the end of the proof of Theorem 2.8 $\operatorname{Tor}_{i}^{R}(S, M)=0$ for all $i \geq 1$ whenever $n$ successive terms of $\operatorname{Tor}^{R}(S, M)$ vanish. This holds since $\operatorname{pd}_{R}(S)<\infty$, which shows that the following sequence of $S$-modules:

$$
0 \rightarrow S \otimes_{R} M \rightarrow S \otimes_{R} P_{n} \rightarrow \cdots \rightarrow S \otimes_{R} P_{1} \rightarrow S \otimes_{R} M \rightarrow 0
$$


is exact. Note that each $S \otimes_{R} P_{i}$ is a projective $S$-module.

On the other hand, for any $S$-module $N$, $\operatorname{Ext}_{S}^{i}\left(S \otimes_{R} M, N\right)=\operatorname{Ext}_{R}^{i}(M, N)$ or all $i \geq 1$ (by [5], Proposition 4.1.3]). If the $S$-module $N$ is projective, then it has finite projective dimension as an $R$-module. Indeed, $\operatorname{pd}_{R}(S)<\infty$ implies that $\operatorname{pd}_{R}\left(S^{(I)}\right)<\infty$ for any index set $I$, and so does $N$ being a direct summand of a free $S$-module. Then, $\operatorname{Ext}_{S}^{i}\left(S \otimes_{R} M, N\right)=\operatorname{Ext}_{R}^{i}(M, N)=0$ by the observation above. Therefore, by Theorem $2.8, S \otimes_{R} M$ is an $n$-strongly Gorenstein projective $S$-module.

Corollary 2.11 Let $R \rightarrow S$ be a homomorphism of commutative rings with $\operatorname{pd}_{R}(S)<\infty$. If $M$ is a Gorenstein projective $R$-module, then $S \otimes_{R} M$ is a Gorenstein projective $S$-module.

Proof. Let $M$ be a Gorenstein projective $R$-module. Then, it is a direct summand of a strongly Gorenstein projective $R$-module $G$ (by [3, Theorem 2.7]). Then, $S \otimes_{R} M$ is a direct summand of the $S$-module $S \otimes_{R} G$ which is, from Theorem 2.10 strongly Gorenstein projective. Therefore, from 3. Theorem 2.7], $S \otimes_{R} M$ is a Gorenstein projective $S$-module, as desired.

Finally, it is convenient to mention that one could define and study the $n$-strongly injective and

flat modules as we have done in the Gorenstein projective case, and so Proposition [2.5. Theorems 2.8 and 2.10, and Corollaries 2.9 and 2.11 have the Gorenstein injective and flat counterparts.

Acknowledgements. The authors thank the referee for his/her careful reading of this work.

\section{References}

[1] M. Auslander, Anneaux de Gorenstein et torsion en algèbre commutative, Secrétariat mathématique, Paris, 1967, Séminaire d'algèbre commutative dirigé par Pierre Samuel, 1966/67. Texte rédigé, d'après des exposés de Maurice Auslander, par Marquerite Mangeney, Christian Peskine et Lucien Szpiro, Ecole Normale Superieure de Jeunes Filles.

[2] M. Auslander and M. Bridger, Stable module theory, Memoirs. Amer. Math. Soc. 94 (American Mathematical Society, Providence, Rhode Island, 1969).

[3] D. Bennis and N. Mahdou, Strongly Gorenstein projective, injective, and flat modules, J. Pure Appl. Algebra 210 (2007) 437-445.

[4] D. J. Benson and K. R. Goodearl, Periodic flat modules, and flat modules for finite Groups, Pacific J. Math. 196 (2000) 45-67.

[5] H. Cartan and S. Eilenberg, Homological Algebra, Princeton Mathematical Series 19 (Princeton University Press, Princeton, 1956).

[6] L. W. Christensen, Gorenstein dimensions, Lecture Notes in Math. 1747 (Springer-Verlag, Berlin, Heidelberg, 2000).

[7] L. W. Christensen, A. Frankild, and H. Holm, On Gorenstein projective, injective and flat dimensions - a functorial description with applications, J. Algebra 302 (2006) 231-279.

[8] E. E. Enochs and O. M. G. Jenda, Relative homological algebra, de Gruyter Expositions in Mathematics 30 (Walter de Gruyter \& Co., Berlin, 2000).

[9] E. Enochs and O. Jenda, Gorenstein injective and projective modules, Math. Z. 220 (1995) 611-633. 
[10] E. E. Enochs and O. M. G. Jenda, On Gorenstein injective modules, Comm. Algebra 21 (1993) 3489-3501.

[11] E. Enochs, O. Jenda and B. Torrecillas, Gorenstein flat modules, Nanjing Daxue Xuebao Shuxue Bannian Kan 10 (1993) 1-9.

[12] E. Enochs, O. Jenda and J. Xu, Foxby duality and Gorenstein injective and projective modules, Trans. Amer. Math. Soc. 348 (1996) 3223-3234.

[13] H. Holm, Gorenstein homological dimensions, J. Pure Appl. Algebra 189 (2004) 167-193.

[14] J. J. Rotman, An Introduction to Homological Algebra (Academic Press, London, New York, 1979). 